\title{
RDUS
}

Revue de DROIT

UNIVERSITÉ DE SHERBROOKE

Titre : $\quad$ CONTRATS ENTRE NON-PRÉSENTS ET CONTRATS ENTRE PRÉSENTS :

Y A-T-IL UNE DIFFÉRENCE?

Auteur(s): $\quad$ Serge GAUDET

Robert P. KOURI

Revue : $\quad$ RDUS, 1989-1990, volume 20, numéro 1

Pages: $\quad 175-199$

ISSN : $\quad 0317-9656$

Éditeur : $\quad$ Université de Sherbrooke. Faculté de droit.

URI : $\quad$ http://hdl.handle.net/11143/13548

DOI : $\quad$ https://doi.org/10.17118/11143/13548 
Page vide laissée intentionnellement. 


\title{
Chronique de droit civil
}

\section{CONTRATS ENTRE NON-PRÉSENTS ET CONTRATS ENTRE PRÉSENTS: Y A-T-IL UNE DIFFÉRENCE?}

\author{
par Serge GAUDET* \\ Robert P. KOURI ${ }^{* *}$
}

Par ce commentaire, les auteurs discutent des différentes solutions mises de l'avant par la doctrine et la jurisprudence afin de déterminer le moment et le lieu de formation de contrat entre nonprésents. Il est souligné que les solutions suggérées sont souvent contradictoires. Or, selon eux qu'il s'agisse d'un contrat inter absentes formé par correspondance ou par moyen de communication instantanée, ou d'un contrat entre présents, la solution devrait être toujours identique: le contrat est conclu lorsque l'offrant a connaissance actuelle ou présumée de l'acceptation.

In this commentary, the authors discuss the various solutions suggested by doctrine and jurisprudence in determining the moment and the place at which contracts between persons not in the presence of one another are formed. It is noted that the solutions retained are often contradictory. It is suggested that in fact, whether one is dealing with a contract inter absentes, entered into by correspondance or by instantaneous means of communication, or with a contract formed between persons in the presence of one another, the solution is identical: a contract is concluded when the offeror has knowledge, actual or presumed, of its acceptance.

\footnotetext{
*. $\quad$ Avocat, Clarkson, Tétrault, Montréal.

**. Professeur, Faculté de droit, Université de Sherbrooke.

Les auteurs tiennent à remercier Me Nathalie Grégoire pour sa précieuse collaboration au niveau de la recherche.
} 


\section{SOMMAIRE}

INTRODUCTION .................. 177

I. Le processus d'accord de volontés dans les contrats entre nonprésents lorsque les parties utilisent un ou des moyens de communication qui ne sont pas instantanés ........ 178

A) Solutions doctrinales $\ldots \ldots \ldots \ldots \ldots \ldots \ldots \ldots \ldots$

i) L'accord de volontés comme coexistence de deux volontés ................... 179

ii) L'accord de volontés comme étant la rencontre de deux volontés ................. 181

B) La solution jurisprudentielle: la théorie de la réception ........................ 184

i) La première époque: l'exigence de la communication de l'acceptation ......... 185

ii) La deuxième époque: un renversement de la jurisprudence ............... 186

iii) Troisième période: retour aux origines . . . . . 188

II. Processus d'accord de volontés dans les contrats entre nonprésents lorsque un ou des moyens de communication instantanés sont utilisés . . . . . . . . . . . . . . . . 193

III. Le processus d'accord de volontés dans les contrats entre présents ......................... 196

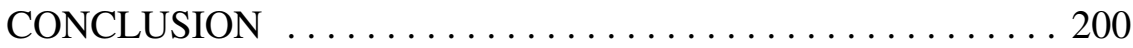




\section{INTRODUCTION}

La réponse à la question qui tient lieu de titre à cette chronique paraîtra évidente à quiconque a étudié le problème du processus de l'accord de volontés dans la formation des contrats. En effet, l'on se hâtera de répondre que la différence est de taille.

D'un côté, le processus de l'accord des volontés, lorsque les contractants sont en présence l'un de l'autre, est tout aussi simple qu'universellement admis: le pollicitant fera une offre qui, si elle rencontre les critères de fermeté et de précision requis, mènera à la conclusion d'un contrat dès que l'acceptant aura manifesté sa volonté de l'accepter.

D'un autre côté, le processus de l'accord de volontés, lorsque les contractants ne sont pas en présence l'un de l'autre ${ }^{1}$, est loin d'être aussi simple et de faire l'unanimité. D'une part, des arrêts et des auteurs soutiennent qu'un tel contrat ne sera définitivement conclu que lorsque l'offrant a eu une connaissance réelle ou présumée de l'acceptation. D'autres argumentent au contraire que ces contrats seraient conclus, soit lorsque se manifeste la volonté d'accepter de l'acceptant, soit lorsque ce dernier fait parvenir son acceptation à l'offrant.

Bref, alors que tout serait simple et admis en ce qui concerne le processus de l'accord de volonté entre présents, tout semble voué à une éternelle complexité en ce qui concerne les contrats entre non-présents.

Or, nous croyons que cette différence n'existe pas en réalité: nous croyons que les mêmes règles s'appliquent que les contractants soient ou non en présence l'un de l'autre. De plus, et c'est cette prétention qui sera sans doute la plus surprenante, contrairement à ce qui semble admis par la doctrine et la jurisprudence, tant en France qu'au Québec, nous croyons que même dans les contrats entre présents, ce n'est pas l'acceptation d'une offre qui fait naître le contrat mais que celui-ci n'est parfait que lorsque l'offrant a connaissance de l'acceptation de son offre. Nous tenterons donc de démontrer que l'acceptation, dans tous les cas, ne clôt pas l'accord de volontés mais n'est que la seconde étape d'un processus qui en compte une troisième, la connaissance de l'acceptation par l'offrant.

1. Evidemment, il ne faut pas confondre le contrat entre non-présents et le contrat conclu par l'intermédiaire d'un mandataire puisque dans ce dernier cas, il s'agit bel et bien d'un contrat négocié par des personnes en présence l'une de l'autre, même si les parties ne le sont pas. 
Ce faisant, nous espérons simplifier grandement, en les rendant pour la plupart non pertinents, les problèmes de plus en plus complexes et insolubles auxquels se heurtera nécessairement le droit québécois s'il persiste à établir une distinction entre les accords de volontés qui prennent place entre présents ou entre non-présents.

Notre démonstration se fera en trois étapes. Dans un premier temps, nous nous attarderons à démontrer qu'en matière de contrats entre nonprésents où l'on utilise un moyen de communication qui n'est pas instantané ${ }^{2}$, le droit québécois a finalement opté pour une conception de l'accord de volonté qui exige que l'offrant ait une connaissance, réelle ou présumée, de l'acceptation afin de parfaire l'entente. Ensuite, nous verrons qu'en ce qui concerne les contrats entre non-présents lorsqu'un moyen de communication instantané est utilisé (v.g. contrats par téléphone), le droit québécois considère que l'instantanéité du moyen de communication rendant ces contrats similaires à ceux conclus entre présents, l'accord de volontés est complet par la seule acceptation. Finalement, nous tenterons de démontrer, à l'aide d'exemples, qu'aucune raison n'existe pour traiter de façon différente le processus de l'accord des volontés dans les contrats entre présents de celui régissant les contrats entre non-présents et qu'ainsi la distinction entre les moyens de communication instantanés et non-instantanés n'a pas de raison d'être.

I. Le processus d'accord de volontés dans les contrats entre nonprésents lorsque les parties utilisent un ou des moyens de communication qui ne sont pas instantanés

Afin de bien comprendre la position qu'a adoptée en cette matière la jurisprudence québécoise, il est utile de se rappeller les diverses solutions qu'a élaborées la doctrine.

\section{A) Solutions doctrinales}

La doctrine se divise en deux camps principaux qui s'opposent sur la notion même de ce que constitue un véritable «accord» de volontés. Chacun de ces camps se divise au sujet de deux solutions, la deuxième solution étant une atténuation de la première pour des raisons d'ordre pratique. Il y a donc quatre possibilités en tout mais qui doivent être étudiées par paires.

2. Nous entendons par «moyen de communication instantané», tout mode de transmission d'une information pour lequel le laps de temps entre l'envoi du message par l'émetteur et sa réception par le récepteur est négligeable. L'exemple le plus typique est la conversation téléphonique. 
i) L'accord de volonté comme coexistence de deux volontés

Pour les tenants de cette première école, l'accord de volonté n'est rien de plus que la simple coexistence de deux volontés portant sur le même objet. Les personnes dont émanent ces volontés n'ont pas à connaître la volonté de l'autre: pour qu'il y ait «accord» de leur volontés respectives, il faut mais il suffit que celles-ci portent au même moment sur la même chose. Dans ce cas, le contrat se sera formé au moment et à l'endroit où l'acceptant manifeste, en l'extériorisant, sa volonté d'accepter. On l'appelle donc la théorie de la déclaration ${ }^{3}$.

Cette théorie n'est pas sans séduire, car elle se base tout simplement sur une application littérale de la description classique ${ }^{4}$ du processus de l'accord de volonté dans le cas des contrats entre présents: dès l'acceptation, le contrat est conclu puisqu'il y a dès lors accord de volonté. Elle comporte néanmoins deux faiblesses.

La première concerne le droit de la preuve: la théorie de la déclaration met l'offrant à la merci de l'acceptant en ce qui concerne la preuve de la conclusion du contrat. En effet, il est fort possible qu'après avoir manifesté son intention d'accepter (par exemple, en écrivant qu'il accepte l'offre), l'acceptant change d'idée et désire ne plus être lié par le contrat. Il n'aura qu'à détruire le papier sur lequel il avait extériorisé son acceptation pour que l'offrant ne sache jamais que le contrat était bel et bien conclu.

Pour rétablir un certain équilibre entre les contractants, il faut apporter une modification à la théorie de la déclaration. De façon à ce que l'offrant puisse obtenir lui aussi une preuve de la conclusion du contrat, posons que le contrat ne sera parfait que lorsque l'acceptant se dessaisira de son acceptation en l'expédiant, par quelque moyen que ce soit, à l'offrant. Ainsi, l'offrant, tôt ou tard, devant normalement recevoir la preuve de la manifestation de volonté de l'acceptant, ce dernier ne pourra que difficilement nier celle-ci. C'est la théorie de

3. G. Marty et P. Raynaud, Droit civil-Les obligations, t.I, $2^{\mathrm{e}}$ éd., Paris, Sirey, 1988, no. 117 à 124, pp. 114 à 122; sans être aussi catégorique R. Beudant, Cours de droit civil français, Paris, Rousseau \& Cie, 1936, no. 84-92, pp. 60-66, semble néanmoins sympathique à cette thèse. M. Laborde-Lacoste, Exposé méthodique de droit civil, t.2, Paris, Sirey, 1947, no. 91, pp. 37-38; G. Baudry-Lacantinerie et L. Barde, Traité théorique et pratique de droit civil, t.XII, Paris, Sirey, 1906, no. 37 à 40, pp. 56-69; C. Demolombe, Cours du Code Napoléon, t.24, Paris, Imprimerie Générale, 1877, no. 6175, pp. 57-78;

4. Mais erronée selon nous, voir infra aux pp. 182-183. 
l'expédition ${ }^{5}$. Ce qu'il faut noter, c'est que cette dernière théorie, tout en étant différente de celle de la déclaration, lui ressemble sur un point fondamental: elle aussi partage la conception d'un «accord» de volonté comme n'étant que la simple coexistence de deux volontés portant sur le même objet. En effet, avec la théorie de l'expédition, le contrat sera conclu avant que l'offrant sache que son offre a été acceptée.

C'est là la deuxième faiblesse de la théorie de la déclaration, qui est donc aussi celle de la théorie de l'expédition,: elle conçoit l'accord de volontés comme ne nécessitant nullement une rencontre de volontés. L'on peut ainsi être lié par un contrat sans savoir que son offre a été acceptée. Peut-on alors parler d'un «accord» de volontés? Comme l'a justement remarqué Gaudemet:

«[C]e qui importe, c'est de savoir ce que les parties entendent par concours de volontés produisant des effets juridiques. Nous venons de voir que ce n'est pas le concours métaphysique de deux volontés dont l'une ignore l'autre, c'est le concours conscient de deux volontés qui, réciproquement, se connaissent» ${ }^{6}$.

C'est cette seconde critique, beaucoup plus fondamentale, qui a amené certains à proposer une autre thèse.

ii) L'accord de volontés comme étant la rencontre de deux volontés

Pour les auteurs qui ont formulé cette autre théorie, le processus de l'accord de volontés dont dépend la formation du contrat ne peut se

5. J.B. Duvergier, Le droit civil français, t.1, Paris, Renouard, 1835, pp. 57-58; V. Marcadé, Explications Théoriques et pratiques du Code Civil, t.4, $2^{\mathrm{e}}$ éd., Paris, Delamote, 1873 , no. 395, p. 360; J. Valéry, Des contrats par correspondance, Paris, 1895, passim; C. Lyon-Caen et L. Renaud, Traité de droit commercial, t.3, $5^{\mathrm{e}}$ éd., Paris, L.G.D.J., 1923, no. 25-28, pp. 22-29; A. Colin et H. Capitant, Droit civil français, t.2, $4^{\mathrm{e}}$ éd., Paris, Dalloz, 1924, pp. 273-74; C. Bufnoir, Propriété et contrat, $2^{\mathrm{e}}$ éd., Paris, Rousseau, 1924, pp. 469-71; L. Josserand, Cours de droit civil positif français, t.II, $3^{\mathrm{e}}$ éd., Paris, Sirey, 1939, no. 51-54, pp. 32-36; H.L. et J. Mazeaud et F. Chabas, Leçons de droit civil, t.II, Vol.I, $7^{\mathrm{e}}$ éd. par F. Chabas, Paris, Montchrestien, 1985, no. 139 à 146, pp. 126-133; J. Ghestin, Traité de droit civil, t.2, 2 éd., Paris, L.G.D.J., 1988, no. 243 à 256-1, pp. 272-281; H. Turnbull «Revocability of acceptance in correspondence contracts» (1930) 8 C.B.R. 615; E. Vipond, «Obligations arising from contracts», (1945) R du B. 472-76; A. Kahn, «Contracts by correspondence», (1959-60), 6 McGill L.J. 98.

6. E. Gaudemet, Théorie générale des obligations, Paris, Sirey, 1965, pp. 47-48. 
concevoir autrement que par une véritable rencontre de volontés: la simple coexistence de deux volontés n'est pas suffisante: pour qu'il y ait «accord» de volontés, ces dernières doivent se rencontrer en un lieu et à un moment donnés et le contrat ne saurait être considéré conclu avant que cette rencontre n'ait eu lieu. En conséquence, le contrat ne sera parfait qu'au lieu et au moment où l'offrant prendra connaissance de l'acceptation: c'est la théorie de l'information?

A l'instar de la théorie de la déclaration, celle de l'information met également l'un des contractants à la merci de l'autre. Alors que pour les raisons déjà mentionnées, la théorie de la déclaration met l'offrant à la merci de l'acceptant, celle de l'information renverse les rôles. En effet, puisque le contrat ne sera pas conclu tant que l'offrant n'aura pas pris connaissance de l'acceptation, le pollicitant ayant changé d'idée quant à l'opportunité de conclure l'entente n'aura qu'à éviter de prendre connaissance de l'acceptation pour que le contrat ne puisse naître. Par exemple, il n'ouvrira pas la lettre que l'acceptant a postée ou il refusera d'écouter les messages qu'aurait pu laisser sur son répondeur l'acceptant. Et même s'il le faisait, la preuve de ce fait demeurerait toujours pour le moins problématique.

Encore une fois, ces considérations de preuve obligent à apporter à la théorie de l'information une atténuation de façon à remettre sur un pied d'égalité nos deux contractants. Présumons donc que l'offrant prend connaissance de l'acceptation dès qu'il a la possibilité d'en prendre connaissance, c'est à dire dès qu'il l'aura reçue. La théorie de la réception décide donc que le contrat est conclu au lieu et au moment où l'offrant a reçu l'acceptation, puisque celui-ci est alors présumé en avoir dès lors pris connaissance ${ }^{8}$.

7. C. Toullier, Le droit civil français, t.6, $2^{\mathrm{e}}$ éd., Paris, Warée, 1819, p. 32; M. Troplong, De l'échange et du louage, Bruxelles, Méline, Cans et Cie, 1841, no. 105, p. 69; M.L. Larombière, Traité théorique et pratique des obligations, t.I, Paris, 1885, no. 21, pp. 1822; F. Laurent, Principes de droit civil, t.XII, $4^{\mathrm{e}}$ éd., Paris, éd. B.C. et C., 1887, no. 479, pp. 550-53; A. Hudelot et E. Metman, Des obligations, Paris, Sirey, 1908, no. 45, pp. 35-37; R. Demogue, Traité des obligations, t.II, Paris, éd. Rousseau, 1923, no. 572580, pp. 212-231; M. Planiol et G. Ripert, Traité pratique de droit civil français, t.VI, Obligations, par P. Esmein, Paris, L.G.D.J., 1952, no. 155-165, pp. 182-198; F. Langelier, Cours de droit civil, t.3, Montréal, Wilson et Lafleur, 1907, p. 373; P.B. Mignault, Le droit civil canadien, t.5, Montréal, éd. Théorêt, 1901, p. 198.

8. M. de Labarre, «La formation du consentement» in La formation du contrat - L'avantcontrat, t.I, Paris, Imp. A. Rey, 1964, pp. 74-80; E. Gaudemet, op.cit. note 6, pp. 42-51; P. Guiho, Cours de droit civil, t.4, Lyon, L'Hermès, 1979, no. 47-52, pp. 34-36; C. Larroumet, Droit civil - Les obligations, t.III, Paris, Economica, 1986, no. 276 à 287, pp. 244-251; P. Magnan, «Du contrat par correspondance», (1957-58) 8 Thémis 20. 
Ce qu'il faut noter c'est que, tout comme les tenants des théories de l'expédition et de la déclaration partagent la même notion de l'«accord de volontés» comme étant une simple coexistence de celles-ci, ceux des théories de la réception et de l'information, au contraire, estiment que le contrat n'est conclu que lorsque l'offrant a eu une connaissance, réelle ou présumée, de l'acceptation. Plus qu'une coexistence de volontés, il y a dans ce dernier cas, une véritable rencontre de volontés.

Les partisans de l'accord de volontés comme simple coexistence n'ont pas manqué de critiquer ceux qui prétendent que la rencontre de volontés est la condition sine qua non de la conclusion du contrat. Selon eux, lorsque les contractants ne sont pas en présence l'un de l'autre, il est impossible qu'il y ait véritablement «rencontre» de volontés. En effet, lorsque l'offrant apprend que l'acceptant a accepté, l'acceptant, lui, ne sait pas que l'offrant sait qu'il a accepté... et ainsi de suite ad infinitum. Il n'y aura donc, dans ces cas, jamais de véritable rencontre de deux volontés qui se connaissent mutuellement ${ }^{9}$.

Cet argument, malgré son élégance, ne nous convainc pas. En effet, il y a tout un monde de différence entre l'incertitude de l'offrant qui ne sait pas si son offre a été acceptée et celle de l'acceptant qui, bien que n'étant pas certain que son acceptation est connue de l'offrant, a néanmoins toutes les raisons de croire que celle-ci le sera tôt ou tard. En d'autres termes, la conclusion du contrat ne saurait constituer une surprise pour l'acceptant, alors que pour l'offrant, elle demeure intrinsèquement imprévue puisqu'il ne sait pas si l'acceptant acceptera ${ }^{10}$.

Aussi, la critique de la régression à l'infini n'est pas valable puisqu'elle ne tient pas compte de la différence de nature qui sépare l'ignorance de l'offrant de celle de l'acceptant. Dans le premier cas, l'offrant ignore l'existence même de la volonté de l'acceptant, alors que l'acceptant, conscient de la volonté de l'offrant et, évidemment, de la sienne propre, a une connaissance personnelle de ces deux volontés. Décider que le contrat ne sera conclu que lorsque l'offrant aura connu (ou du moins qu'il aura reçu) l'acceptation, c'est uniquement faire en sorte que le contrat ne soit définitivement conclu qu'au moment où

9. J. Valéry, op.cit. note 5, p. 161; L. Josserand, op.cit. note 5, no. 53, p. 34; V. Marcadé, op.cit. note 5, no. 395, p. 360; G. Baudry-Lacantinerie et L. Barde, op.cit. note 3, no. 37, p. 59; H.L. et J. Mazeaud et F. Chabas, op.cit. note 5, no. 146, p. 131-32.

10. G. Masse, Le droit commercial dans ses rapports avec le droit des gens et le droit civil, t.3, $2^{\mathrm{e}}$ éd., Paris, Durand, 1862, no. 1453, p. 34; G. Baudry-Lacantinerie et L. Saignat, Traité théorique et pratique du droit civil: De la vente et de l'échange, $3^{\mathrm{e}}$ éd., Paris, Larose, 1908, no. 36, pp. 27-28; R. Demogue, op.cit. note 7, no. 576-77, pp. 219-23; E. Gaudemet, op.cit. note 6, p. 48. 
l'offrant aura lui aussi le bénéfice de connaître les deux volontés qui, ensemble, vont faire naître le contrat.

On le voit bien: la théorie de l'information, et sa version pragmatique, la théorie de la réception, mettent ainsi sur un pied d'égalité l'offrant et l'acceptant à propos de ce qui compte vraiment: la connaissance de l'existence des deux volontés créatrices du contrat. Ce n'est donc qu'à compter de ce moment que l'on peut parler de la rencontre de deux volontés qui se connaissent.

Le choix est donc simple: pratiquement l'on optera soit pour la théorie de l'expédition si notre conception de l'accord de volontés n'en fait que deux volontés qui coexistent ou soit pour la théorie de la réception, si l'on conçoit plutôt celui-ci comme une rencontre de volontés ${ }^{11}$.

C'est cette dernière thèse que nous retenons et c'est celle, à notre avis, qu'a retenue la jurisprudence québécoise, tel que nous le verrons dans la prochaine section.

B) La solution jurisprudentielle: la théorie de la réception

Le choix de la théorie de la réception par nos tribunaux ne s'est pas fait sans difficultés. Trois périodes sont à distinguer.

11. Notons que ce choix n'est aussi simple que dans la mesure où l'on accepte que la seule conception de l'accord de volontés doit résoudre toutes les questions: c'est ce qu'on a appellé l'attitude moniste. Toutefois, étant donné les nombreuses difficultés que posent le problème des contrats entre non-présents, certains auteurs français ont adopté une attitude dualiste ou même parfois pluraliste du problème: la solution variera selon le problème à résoudre (moment de formation? lien de formation? compétence juridictionnelle? possibilité de rétractation? etc...) Voir, J.L. Aubert, Notions et rôles de l'offre et de l'acceptation dans la formation du contrat, Paris, L.G.D.J., 1970, no. 410-420, pp. 378-392; Ph. Malaurie, note sous Civ. $1^{\text {ere }}, 21$ décembre 1960, D.1961.417; J. Flour et J.L. Aubert, Les obligations, Vol.I, Paris, éd. A. Colin, 1975, no. 162-178, pp. 112-122; B. Starck, Droit civil: obligations, t.II, $2^{\mathrm{e}}$ éd., Paris, Litec, 1986, no. 287-318, pp. 100-110; Ph. Malaurie et L. Aynès, Cours de droit civil: les obligations, Paris, éd. Cujas, 1985, no. 255-56, pp. 184-187. Au contraire du droit français, le droit québécois n'a pas cru bon de dissocier la solution de ces divers problèmes, il a toujours tenté de les régler uniquement par la notion de l'accord de volontés, il a donc adopté une attitude moniste que nous n'entendons pas critiquer. Les thèse dualistes et pluralistes sont aussi complexes qu'encombrantes et tombent finalement dans une casuistique peu conciliable avec la théorie juridique. Il y a dans l'attitude moniste une simplicité et un souci de cohérence que nous ne pouvons qu'approuver. 
i) La première époque: l'exigence de la communication de l'acceptation

Après quelques faux départs ${ }^{12}$, la jurisprudence québécoise opte pour l'exigence de la communication de l'acceptation à l'offrant. Dans Underwood v. Maguire ${ }^{13}$, le juge Wurtele, écrivant pour ses collègues Baby et Blanchet, était d'avis que:

«It is... necessary for the completion of an agreement that the acceptance of the offer has become known to him who made it (...). The contract is only formed at the moment when both parties are aware that they have mutually consented to an agreement based on the proposition.

(...)

To form a contract, it is not sufficient that the will of both parties should co-exist, it is necessary that there should be a concurrence or joint accord, and for this concurrence to exist it is necessary that the proposer should know that his offer has been accepted, so that there should be a blending of intention at a given moment...» ${ }^{14}$

Toutefois, les juges Hall et Bossé différaient d'avis. Ce dernier, écrivant au nom des deux, fait mention de la doctrine divisée en France, mais ajoute que, selon lui, la théorie de l'expédition est plus conforme aux intérêts du commerce, la preuve en étant que «la jurisprudence en Angleterre et aux Etats-Unis, les deux plus grands pays commerciaux du monde, est maintenant fixée en ce sens» ${ }^{15}$.

12. Clark c. Ritchey (1863), 9 L.C.J. 234; Gannon c. Sauvé (1887), 10 L.N. 211; Gratton c. Brennan (1887), 15 R.L. 713. Ces arrêts, aux faits similaires, décident que lorsque des commandes sont acceptées et, qu'en conséquence, le marchand fait livrer les marchandises, le contrat est conclu au lieu et au moment où s'est produit cette acceptation telle que manifestée par l'envoi des marchandises.

13. (1895), [1897] 6 B.R. 237 (ci-après «Underwood»).

14. Ibid. à la p. 247.

15. Ibid. à la p. 244. 
La jurisprudence québécoise allait, par la suite, se conformer à la décision majoritaire de la Cour d'appe ${ }^{16}$.

ii) La deuxième époque: un renversement de la jurisprudence

Avec l'affaire Magann c. Auger ${ }^{17}$, la Cour suprême, pour la première fois, avait à se pencher sur la question de la formation du contrat inter absentes. Une offre ayant été postée de Québec à Toronto, l'acceptation fut postée de Toronto à Québec. Le contrat avait-il été conclu à Toronto ou à Québec? Cette question se soulevait à l'occasion d'une exception déclinatoire, l'action ayant été intentée à Québec.

La Cour supérieure et la Cour d'appel rejettèrent l'exception déclinatoire au motif que par ses actes, le défendeur s'était volontairement soumis à la juridiction du tribunal. Rejetant cette prétention, le juge Taschereau, rendant le jugement de la Cour suprême, eût donc à se prononcer sur le fond de la question.

Il suivit alors à la trace le raisonnement du juge Bossé, dissident dans Underwood. Il commence par expliquer que, quoique la doctrine majoritaire en France opine que l'acceptation doit avoir été communiquée à l'offrant pour que l'on puisse considérer le contrat conclu, il lui semble que le raisonnement des auteurs minoritaires est plus logique. Il résume ainsi leur argument principal:

«If it were required for the aggregatio mentium necessary to create mutuality of obligations in a contract made by correspondence that the party who has made the offer has received the acceptance of his offer, it would follow that the party accepting should himself not be bound till he is informed that his acceptance has reached the party offering. It is obviously of the greatest importance to the commercial community that such a doctrine should not prevail» ${ }^{18}$.

Et, il ajoute, dévoilant probablement là le véritable motif de la décision de la Cour:

16. McFee c. Gendron (1889), 18 R.L. 230; Beaubien Produce \& Milling Co. c. Robertson (1900), 18 C.S. 429; Hislop c. Bernatz (1901), 3 R.P. 451; Reeves c. McCullock (1901), 4 R.P. 285.

17. (1901), 31 R.C.S. 186 (ci-après «Magann»).

18. Ibid., à la p. 193 . 
«We declare the law to be in the Province of Quebec upon the same footing as it stands in England, and in the rest of this dominion...» ${ }^{19}$

Le renversement par rapport à Underwood était complet. La jurisprudence québécoise, non sans quelques réticences ${ }^{20}$, se plia à la nouvelle conception de l'accord de volontés que lui imposait la Cour suprême $^{21}$. Comme l'a écrit W.F. Chipman, après l'affaire Magann,:

«We may now take it as the law all over Canada that, in any contract by correspondence, whether letter or telegram be the mode of transmission, (...) the acceptor has bound both himself and the offeror when he has dispatched the expression of his will» ${ }^{22}$.

iii) Troisième période: retour aux origines

En 1927, nouveau coup de théâtre. Dans Charlebois c. Baril (ciaprès Charlebois $)^{23}$, une offre fut envoyée par messager et l'acceptation fut postée. Après que cette acceptation fut mise à la poste mais avant qu'elle arrive à destination, une révocation de l'offre parvient à l'acceptant. Y-avait-il eu conclusion du contrat avant cette révocation? La Cour d'appel, appliquant les principes de l'affaire Magann décida que le contrat s'était conclu dès la mise à la poste de l'acceptation ${ }^{24}$. La Cour suprême, infirma cette décision et prit résolument parti pour la théorie de la réception. Le juge Anglin, parlant au nom de ses collègues Mignault, Newcombe, Rinfret et Lamont, va même jusqu'à écrire que l'arrêt Magann ne dit pas vraiment ce qu'il semble vouloir dire. Contrairement à ce que toute la jurisprudence a compris, l'arrêt Magann

19. Ibid., à la p. 193.

20. Voir, les réticences, ou même le refus total, de certains juges d'appliquer cette nouvelle conception de l'accord de volontés: Ward c. Johnston (1903), 5 R.P, 123; Borgfield c. La Banque d'Hochelaga (1905), 28 C.S. 344; Butler c. Mechanical Equipment Co. of Canada (1912), 22 K.B. 530; Fecteau c. McLandress (1921), 59 C.S. 470.

21. Ward c. Johnston, supra, note 20; Schmidt c. Crowe (1903), 5 R.P. 361; Timossi c. Palangio (1904), 26 C.S. 70; United Shoe Co. c. Caron, (1904), 11 R. de J. 59; Beaudoin c. Watterson (1910), 19 K.B. 530; Butler c. The Mechanical Equipment Co. of Canada, supra, note 20; Paquet c. Balcer (1913), 44 C.S. 36; Bell c. Chase \& Co. (1916), 22 R.L.n.s. 438; Laferté c. Martel (1917), 19 R.P. 249; Gagnon c. Labrecque (1919), 25 R.L.n.s. 376; Sherwin c. Quebec Fish and Fruit Exchange Ltd. (1925), R.P. 387 (B.R.); Charlebois c. Baril (1926), 43 C.B.R. 295.

22. «Some Aspects of Business by Telegrams», (1908) 28 Canadian Law Times 817, à la p. 823.

23. (1927), [1928] R.C.S. 88

24. Charlebois c. Baril, supra, note 21, p. 310. 
consacrerait, en fait, la théorie de la réception. Il est utile de citer au long la partie la plus importante du jugement:

«The courts below (...) regarded the judgment of this Court in Magann v. Auger as determining that the mailing of the plantiff's letter of acceptance to the defendant constituted communication of it to him.

With great respect this is an erroneous view of the scope and effect of the decision of this Court. That case was one of contract by correspondence, i.e., the offer was sent by mail and that was held to constitute a nomination by the sender of the post office as his agent to receive the acceptance for carriage to him. (...) But this decision has no application to a case where the offer is communicated, as here, not by mail, but by another means. To make a contract the law requires communication of offer and acceptance alike either to the person for whom each is respectively intended, or to his authorized agent ${ }^{25}$.

Ce qu'il faut noter de ce passage, c'est qu'il renferme trois idées connexes mais distinctes. La première, qui est le principe général, c'est que le contrat n'est pas conclu tant que l'acceptation n'a pas été communiquée à l'offrant. La deuxième, qui est une simple application du mandat, c'est que l'acceptation peut être reçue par l'offrant lui-même ou par un tiers à qui il a confié, expressément ou implicitement, le mandat de recevoir l'acceptation en son nom. A ce moment-là, la réception de l'acceptation par le mandataire vaut évidemment réception par l'offrant lui-même: qui agit per alium agit per $\mathrm{se}^{26}$. La troisième, c'est qu'à moins de stipulation au contraire, l'offrant est présumé nommer la personne qu'il utilise pour faire parvenir son offre à l'acceptant comme mandataire pour recevoir, en son nom l'acceptation.

Donc, en toute hypothèse, il faudra que l'acceptation ait été reçue par l'offrant (ou par son agent, exprès, implicite ou présumé) pour que l'accord de volontés soit complété. Au niveau des principes, l'arrêt Charlebois est donc bel et bien un retour à la décision majoritaire dans Underwood et consacre donc, dans notre droit, la thèse de la rencontre

25. Charlebois, supra, note 23, p. 89.

26. Art. 1727 C.c.B.-C. 
des deux volontés qui se connaissent en rejetant celle des volontés qui ne font que coexister. L'on aura inventé la notion d' «agent présumément autorisé» pour tenter de réconcilier, a posteriori, les affaires Magann et Charlebois. Dans Magann, si la Cour a décidé que le contrat s'était conclu lorsque l'acceptation fut mise à la poste, c'est uniquement parce que l'offre ayant été envoyée par la poste, l'offrant était ainsi présumé avoir mandaté le service canadien des Postes pour recevoir, en son nom, l'acceptation. Aussi, dès que l'acceptation était mise à la poste, elle était reçue par le mandataire de l'offrant. C'est cette réception de l'acceptation par l'offrant, par le biais de son mandataire, qui complétait le processus de l'accord des volontés.

Cette relecture de Magann indique donc bel et bien que la Cour suprême, dans Charlebois, a voulu adopter la théorie de la réception comme principe général. Par ailleurs, elle ne peut masquer le fait que Charlebois opère le renversement complet de la thèse défendue dans Magann. Contrairement à ce qu'en dit le Juge Anglin dans l'affaire Charlebois, il est impossible de lire autre chose dans les motifs du Juge Taschereau dans Magann qu'un refus pur et simple de la thèse voulant que l'accord de volontés se complète par la connaissance par l'offrant de l'acceptation $^{27}$.

Après Charlebois, la jurisprudence québécoise a immédiatement changé son fusil d'épaule et est, en conséquence, revenue à la notion de l'accord de volontés qu'avait consacrée l'affaire Underwood. Elle a aussi considéré qu'il fallait présumer que dès la réception de l'acceptation par l'offrant, celui-ci était informé de cette acceptation, adoptant donc la théorie de la réception. Elle a donc pris bien soin de vérifier quand cette réception avait eu lieu et si elle n'avait pas été le fait d'un agent, expressément, implicitement ou présumément autorisé par l'offrant ${ }^{28}$.

27. Magann, supra, note 17, p. 193.

28. Renfrew Flour Mills c. Sanschagrin (1928), 45 B.R. 29; Association Pharmaceutique de la Province de Québec c. Eaton Co. (1931), 50 B.R. 482 (voir les opinions des juges Dorion et Rivard, contra: opinion du juge Lafontaine); Gagnon c. Fonderie de StAnselme (1932), 36 R.P. 40; Allaire c. Lamontagne (1933), 72 C.S. 69; Duquette c. Schering (1938), 44 R.L. n.s. 422; Premier Trust Co. c. Turcotte (1938), 64 B.R. 401; Verdun Realties Co. c. Lajoie (1947), [1948] C.S. 148; Poulin c. Regent Lumber Co. (1950), [1951] R.P. 188, inf. par la Cour d'appel sans motifs publiés, [1951] B.R. 782; Canada Concrete Products Inc. c. Ville de Nicolet [1953] R.P. 432; Milinkovich c. Canadian Mercantile Insurance Co. [1960] R.C.S. 830; Clerk Windows Ltd. c. Rubatex Corp. (1964) [1965] R.P. 276; Godin c. Leblanc [1970] C.S. 46; Hunter c. Vandervoore [1972] R.P. 414; In Re Witt: Bissell c. Zwaig, résumée à [1975] C.A. 853; Les Industries Vanox Ltée c. Les Produits Alcan Canada Ltée [1980] C.S. 490. 
On pourrait donc croire que la question était définitivement réglée en faveur de la théorie de la réception. Toutefois, tout n'est pas si simple.

Premièrement, tel que nous l'avons mentionné, l'on a continué à parler de l'application de la théorie de l'expédition même dans les cas où, par application de la thèse de l'agent autorisé, dans les faits on se trouvait à appliquer la théorie de la réception. Cette mauvaise terminologie est sans conséquence dans la mesure où l'on comprend bien que c'est la réception qui compte même lorsqu'elle se trouve à être concomitante avec l'expédition ${ }^{29}$.

Plus sérieuse est la confusion, trop souvent faite, entre l'«agent autorisé» et le moyen de communication utilisé. Il ne faut pas croire en effet, que les arrêts Magann et Charlebois établissent le principe que lorsque le même moyen de communication est utilisé, alors il faut appliquer la théorie de l'expédition alors que lorsque des moyens différents sont utilisés, il faudrait appliquer la théorie de la réception ${ }^{30}$. En effet, tel que démontré ci-dessus, il n'est pas important que le moyen de communication soit le même ou non, il faut plutôt déterminer, si tant est qu'il y en ait une, qui est la personne qui est l'«agent autorisé» de l'offrant pour recevoir en son nom l'acceptation. Or, un moyen de communication n'est pas une personne et, partant, ne peut évidemment pas être nommé mandataire par l'offrant pour recevoir en son nom l'acceptation.

29. Parler, en ce cas, d'application de la théorie de l'expédition est erroné puisque tel que nous l'avons démontré, ce n'est pas l'expédition de l'acceptation qui compte mais bien la réception de celle-ci par l'agent autorisé de l'offrant. La raison de cette confusion réside dans le fait que lorsque l'acceptant accepte via l'agent autorisé, l'expédition de l'acceptation est évidemment concomitante à la réception par l'agent. Toutefois, au niveau des principes, il est important de comprendre que, quoique concomitantes, c'est la réception par l'offrant qui complète le contrat, pas l'expédition par l'acceptant.

30. Pour des exemples de cette interprétation des affaires Magann et Charlebois voir: J-L. Baudouin, Les obligations, $3^{\mathrm{e}}$ éd., Cowansville, Yvon Blais, 1989, no. 113-118, pp. 104-107; J.-G. Bergeron, Les contrats d'assurance, t. 1, Sherbrooke, Editions SEM Inc., 1989, pp. 206-211; J. Ghestin, Le contrat: dans le nouveau droit québécois et en droit français: principes directeurs, Montréal, Institut de droit Comparé, Université McGill, 1982, pp. 145-47; Office de Révision du Code Civil, Rapport sur le Code Civil du Québec, Volume II, t.2, Québec, Editeur officiel, 1977, p.610, cf. infra, note 48; Angers c. Burger Plywood Ltd. [1967] R.P. 378; J.P. Marketing Inc. c. Continental Information System, J.E. 84-589; Simpson-Sears Ltd. c. Sous-Ministre du Revenu [1983] C.P. 126 inf. par Sous-Ministre du Revenu c. Simpson-Sears Ltd. (1986) R.L. 37 (C.A.). 
Ce n'est donc pas le service des postes ou le service télégraphique qui peut être un «agent autorisé», ce ne peut être que la personne, morale ou physique, qui est propriétaire du réseau de communication. Si notre interprétation de l'arrêt Charlebois, est exacte, on est alors face à la simple superposition des principes du mandat à la théorie de la réception ${ }^{31}$, même s'il est vrai qu'il soit très artificiel de présumer que l'offrant nomme mandataire, pour recevoir l'acceptation, en son nom, la personne dont il requiert les services pour envoyer son offre ${ }^{32}$.

31. Cette interprétation est d'ailleurs celle de la très grande majorité des arrêts et des auteurs. Voir en plus des arrêts cités à la note 28, Louis Baudouin, Le droit civil de la province de Québec, Montréal, Wilson et Lafleur, 1953, pp. 663-65; M. Jetté, «L'affaire Colmenares», (1966) 1 R.J.T. 279, 283; A. Kahn, op.cit. note 5, p. 112-13; A. Larouche, Les obligations, t.I, Théorie générale des contrats; quasi-contrats, Ottawa, éd. U. d'Ottawa, 1982, no. 113, pp. 126-28; G. Mure, «Les contrats entre absents: acta est fabula», 1970 R.D.U.S. 159; J. Pineau et D. Burman, Théorie des obligations, 2 éd., Montréal, Thémis, 1988, pp. 78-82.

32. En effet, s'il est possible pour l'offrant de nommer expressément ou implicitement, quelqu'un devant recevoir l'acceptation en son nom, ce qui est un véritable contrat de mandat, il est pour le moins embêtant de considérer que celui qui n'a pour seule fonction de transporter un message, sans, le plus souvent, en prendre connaissance, soit le mandataire de l'offrant pour recevoir l'acceptation en son nom: il y a là confusion certaine entre le «mandat» et le «louage de services». Voir à ce propos l'opinion du juge Dorion dans l'affaire Ass. Pharmaceutique de la Province de Québec c. Eaton, supra, note 28 , p. 485 . Au même effet, M. Tancelin, Des obligations, $4^{\mathrm{e}}$ éd., Montréal, Wilson et Lafleur, 1988, no. 101, p. 56-57; A. Kahn, op.cit. note 5, p. 113 et seq. Tel que précédemment mentionné, nous croyons que cette théorie de l'«agent présumé autorisé» n'a été élaborée que pour cacher le fait que l'arrêt Charlebois venait véritablement renverser l'arrêt Magann sur la question fondamentale de ce qui est nécessaire pour compléter l'accord de volontés. Il n'y a aucune raison de conserver cette notion de nos jours. 
II. Processus d'accord de volontés dans les contrats entre non-présents lorsque un ou des moyens de communication instantanés sont utilisés

La jurisprudence ${ }^{33}$ et la doctrine ${ }^{34}$ décident, de façon quasiunanime $^{35}$, que le contrat conclu par téléphone l'est au lieu et au moment où l'acceptant prononce les paroles d'acceptation. Ainsi, contrairement aux accords de volontés conclus à l'aide de moyens de communication non-instantanés, ceux faits par l'intermédiaire du téléphone seront completés par la seule acceptation.

Or, comment justifier que selon que l'accord de volontés sera conclu par téléphone ou autrement, la communication de l'acceptation à l'offrant sera ou non nécessaire? Comment expliquer que la notion même de ce qu'est véritablement l'accord de volontés varie selon le processus utilisé pour effectuer cet accord? L'on peut tenter de réconcilier cette jurisprudence concernant les contrats conclus par téléphone avec la jurisprudence traditionnelle. Le seul moyen de le faire c'est de considérer le téléphone comme étant un agent autorisé: la «remise» de l'acceptation au service téléphonique étant alors la réception de l'acceptation par l'agent autorisé de l'offrant. Mais, nous l'avons vu cette issue est impossible! Un téléphone ou même un réseau téléphonique, ca ne demeure qu'un appareil qui est donc, de jure, incapable d'être un agent. Et si l'on rétorque que c'est la compagnie qui exploite le réseau téléphonique qui peut être l'agent et non le réseau luimême (ce qui est vrai) alors il faudrait $1^{\circ}$ ) vérifier si l'offrant fait affaires avec la même compagnie que l'acceptant et $2^{\circ}$ ) où exactement les réseaux téléphoniques de ces compagnies se rencontrent ${ }^{36}$ ! Agir autrement, ce sera toujours confondre totalement le moyen de communications avec l'agent autorisé.

33. Paquet c. Balcer (1913), 44 C.S. 36; Sherwin c. Quebec Fish and Fruit Exchange Ltd. [1925] R.P. 387 (B.R.); Dion c. Chalifoux [1949] R.P. 42 (C.S.); Talbot c. Donnacona Paper Co. [1958] R.P. 202 (C.S.); Angers c. Burger Plywood Ltd. [1967] R.P. 378 (C.S.); Upper Canada Place Ltd. c. Classic's Little Books Ltd., C.A.M., 17 mars 1976, no. 555-755; Pisapia Construction Inc. c. Location d'Equipement Chicoutimi C.A.Q. 09-000149-75, 22 mars 1976; Bonavista Fabrics Ltd. c. Rosenthal \& Rosenthal Inc., J.E. 83-781 (C.S.), conf. par [1984] C.A. 52; Sous-Ministre du Revenu c. Simpson-Sears Ltd. (1986) R.L. 37 (C.A.).

34. J.-L. Baudouin, op.cit note 30, no. 117, p. 107; M. Tancelin, op.cit. note 32, no. 103, p. 58; A. Larouche, op.cit. note 31, no. 115, p. 129.

35. Canada Concrete Products Inc. c. Nicolet [1953] R.P. 432; Simpson-Sears Ltd. c. SousMinistre du Revenu [1983] C.P. 126, inf. par [1986] R.L. 37 (C.A.). Voir aussi, J. Pineau et D. Burman, op.cit. note 31, no. 47, p. 73-74.

36. Dans les faits, même si Bell Canada jouit d'un quasi-monopole au Québec, pas moins de 17 autres entreprises fournissent des services téléphoniques dans la province. On ne peut donc pas tenir pour acquis, contrairement à ce que se passe avec la poste, que tout le monde fait affaires avec Bell Canada. 
Inutile d'ajouter que les tribunaux ne se posent pas ces questions-là avant de décider sommairement que les contrats conclus par téléphone, le sont au lieu et au moment où les paroles d'acceptation sont prononcées.

La seule raison, selon nous, pouvant donc rationnellement justifier cette différence réside dans les caractéristiques mêmes des conversations téléphoniques: celles-ci permettent un contact instantané entre les interlocuteurs de la même façon que la conversation normale des parties l'une en présence de l'autre. Ainsi, Josserand a pu écrire:

«On se demande si les contrats conclus par téléphone sont à traiter comme des contrats entre présents ou comme contrats entre absents: d'une part, ils interviennent entre correspondants éloignés l'un de l'autre; mais d'un autre côté, les demandes et les réponses sont transmises et échangées sans trait de temps; c'est une véritable conversation qui s'engage entre les interessés qui, à un moment donné, acquièrent la certitude de la rencontre de leurs volontés. Cette dernière particularité nous paraît décisive; les contrats par téléphone sont à traiter comme conclus entre présents ${ }^{37}$.

Même si ni la doctrine ni la jurisprudence québécoise n'a jamais pu clairement expliquer pourquoi elle considérait que le contrat conclu par téléphone devait obéir à des règles différentes de celles régissant les autres contrats entre non-présents, nous croyons qu'elle l'a fait, et surtout qu'elle continue de le faire, en raison du caractère instantané de la communication entre les contractants ${ }^{38}$.

En effet, le caractère instantané de la conversation téléphonique la rendant semblable à la conversation des personnes en présence l'une de l'autre, le processus de l'accord de volontés du contrat conclu par téléphone, devrait, en toute logique, obéir aux mêmes règles de formation que celles régissant les contrats conclus entre présents. Or, puisque selon une doctrine et une jurisprudence admise, ces derniers seraient conclus par le seul fait de l'acceptation (et non par la

37. L. Josserand, op.cit. note 5, p. 35.

38. Voir arrêts et auteurs cités aux notes 33 et 34 . Les motifs des arrêts sont confus et contradictoires (voir, par exemple, la décision de la Cour d'appel dans Sous-Ministre du Revenu c. Simpsons-Sears Ltd., supra, note 30, passim), tandis que la doctrine québécoise est pour le moins laconique sur le sujet. 
connaissance par l'offrant que son offre a été acceptée) il s'ensuit que le contrat par téléphone serait lui aussi conclu au lieu et au moment de l'acceptation.

Mais, pour être logique, il faudra traiter pareillement tous les accords de volontés obtenus par des moyens de communications permettant un contact instantané ${ }^{39}$. Dès lors, une question surgira inévitablement. Puisque les conditions du processus de l'accord de volontés sont différentes dans les communications instantanées et les communications non-instantanées: il faudra se demander pour chaque moyen de communication s'il est instantané ou non. Le télécopieur («fax») est-il plus près du téléphone que du courrier? et le télex? et les ordinateurs reliés par modem? Avec les progrès rapide de la technologie, cette question risque de devenir rapidement épineuse.

Plus fondamentalement, comment justifier le fait que la notion même de ce qu'est, dans notre droit, l'«accord de volonté» varie selon que le moyen de communication utilisé soit ou non instantané? C'est le concept même de consentement qui est en cause et on ne voit aucune raison pour que ce concept ne soit pas le même dans tous les cas.

A notre avis, toutes ces questions et incohérences pourraient être évitées dans la mesure où la prémisse de base de ce raisonnement est modifiée: même dans les contrats conclus entre présents, l'accord de volontés n'est véritablement complété que lorsque l'offrant sait, ou est présumé savoir, que son offre a été acceptée. C'est là l'objet de notre troisième partie.

III. Le processus d'accord de volontés dans les contrats entre présents

Tel que nous l'avons mentionné dans l'introduction, les auteurs et la jurisprudence affirment de façon lapidaire que les contrats entre présents se forment par l'acceptation pure et simple d'une offre valable ${ }^{40}$. Dans la plupart des contrats formés entre présents, la généralité de ce genre d'énoncé ne suscite aucune difficulté. En règle générale, dans ces situations il n'y a aucun intérêt à déterminer le moment ou le lieu de formation du contrat.

39. Par exemple, A. Larouche, op.cit. note 31, no. 115, p. 129, écrit: «il faut considérer le "télex", de plus en plus employé, comme le téléphone: il s'agit d'une conversation téléphonique écrite».

40. Il nous semble inutile d'appuyer cette proposition tant les autorités sont nombreuses. Le lecteur peut toujours se référer aux ouvrages des doctrines cités aux notes 5,6,7,8 et 34 . 
Mais il demeure non moins vrai que dans certaines situations impliquant des contractants présents, il y a un intérêt pratique à déterminer de façon précise le lieu de formation du contrat ou le moment même de la rencontre des volontés. Ce n'est qu'en vérifiant la prémisse dans ces hypothèses, que nous pourrons en vérifier la validité. Comme l'a justement fait remarquer W.F. Chipman:

«In an ordinary contract where two persons meet face to face, the offer of the one is met on the spot by the acceptance of the other. The whole affair is so simple, in effect, that the offeror has not needed to perceive that there were really two distinct matters involved, firstly, the other's acceptance of his offer, and secondly, his knowledge of that acceptance ${ }^{41}$.

Il s'agit donc d'imaginer des hypothèses où, quoique le contrat soit conclu entre présents, il importe de déterminer avec exactitude le lieu ou le moment de formation de l'entente. Prenons d'abord l'hypothèse d'un contrat en voie de conclusion entre deux personnes habitant la ville frontalière de Rock Island, une municipalité située à cheval sur la frontière séparant le Québec du Vermont. Du côté québécois de la ligne A fait une offre verbale de vente d'un objet quelconque à B qui se trouve à peine quelques pieds de lui mais en territoire américain. Sans se déplaçer B accepte verbalement l'offre telle que faite. Bien qu'il s'agisse certainement d'un contrat entre présents, il n'est pas sans importance, dans cette situation, de déterminer où s'est formé le contrat, ne serait-ce que pour décider la juridiction compétente pour entendre un éventuel litige ou pour déterminer, à défaut de stipulation expresse des parties, la loi applicable au contrat ${ }^{42}$. Celui-ci s'est-il conclu à l'endroit où se trouve l'offrant (Québec) ou celui où se trouve l'acceptant (Vermont)?

Un autre exemple soulève un problème de détermination du moment où le contrat se forme. A offre de vendre à $\mathrm{B}$ pour tel prix, un certain objet. L'offre en question, comportant un délai de réflexion, est stipulé valable jusqu'à midi, le premier du mois. Les deux parties se donnent mutuellement rendez-vous à Montréal, le premier du mois à midi moins le quart aux coins des rues Peel et Ste-Catherine pour que la décision du pollicité soit annoncée à $\mathrm{A}$. Malheureusement, le jour en question, B arrive légèrement en retard et au moment où il exprime à $\mathrm{A}$ son acceptation, ce dernier ne l'entend pas car à cet instant précis, cinq camions du service des incendies de la Ville de Montréal passent près

41. Loc.cit. note 22, p. 817.

42. Art. 8 C.c.B.-C. 
d'eux avec sirènes et klaxons. Lorsque $B$ est enfin en mesure de se faire entendre par A, le délai pour l'acceptation de l'offre est expiré et A se sent libéré de sa proposition. Y a-t-il eu formation du contrat par l'acceptation de B avant l'expiration du délai, alors que le pollicitant n'a pas entendu la réponse en temps utile?

Pour répondre à ces questions, tout comme pour les contrats entre non-présents, il faut nécessairement décider ce qu'est l'accord de volontés. Celui-ci n'est-il que deux volontés qui coexistent (et qui peuvent s'ignorer) ou s'agit-il plutôt d'une rencontre de deux volontés qui se connaissent réciproquement?

Lorsque, dans les contrats entre non-présents, cette question s'est posée, nous avons vu que la jurisprudence québécoise a opté pour la deuxième conception. Pourquoi en irait-il autrement lorsque les contractants se trouvent en présence l'un de l'autre? Puisque les arguments, déjà exposés, en faveur de l'une ou l'autre de ces thèses sont également applicables au contrat entre présents, la rigueur exige que la solution soit la même dans ces deux situations.

D'ailleurs, dans deux arrêts québécois de première instance, décidés en 1901, les juges ont exprimé leur adhésion à ce point de vue. Même s'il s'agissait dans les deux cas de causes traitant de contrats entre absents, il est utile de voir le raisonnement des magistrats.

Dans Hislop c. Bernatz, le juge Mathieu a écrit:

«L'acceptation ne rend le contrat parfait qu'à partir du moment où elle parvient à la connaissance de celui qui a fait l'offre. C'est ainsi que se forme régulièrement le contrat lorsque les deux parties sont présentes. L'acceptant entend ce que veut le pollicitant, et celui-ci entend la réponse qui est faite à ses offres; voilà le vrai concours de volontés, chacune des parties intéressées sachant ce que veut l'autre» ${ }^{43}$.

(nos italiques)

Dans le même sens, le juge Tellier, dans Reeves v. McCullock, a exprimé l'opinion:

43. Supra, note 16, p. 457. 
«[qu']une convention exige non seulement le concours de deux volontés, mais encore leur manifestation réciproque, et que cette manifestation n'existe pour chaque partie que lorsqu'elle lui arrive par les sens dans les conventions verbales, par la réception de la lettre dans les marchés par correspondance; que tant qu'une des parties ignore le concours de volontés d'où résulte la convention, celle-ci n'existe pas pour elle» ${ }^{44}$.

Du côté de la doctrine, Larombière avait également noté que:

«Je vous écris pour vous proposer la vente de ma maison. La convention ne s'achèvera que par votre acceptation connue de ma part (...). Les choses ne se passent pas autrement quand on correspond que quand on s'abouche» ${ }^{45}$.

A la lumière de ce que nos tribunaux ont decidé dans les contrats entre non-présents, nous voyons donc que la solution aux hypothèses soulevées pour illustrer nos propos repose nécessairement sur l'idée que non seulement les deux volontés doivent coexister, mais que cette coexistence doit être connue des deux contractants. Ainsi, dans le premier cas, (celui du contrat conclu à Rock Island) le contrat serait formé au Québec car la réponse est parvenue au pollicitant qui se trouvait en territoire québécois. Dans le deuxième cas, l'offre serait caduque à cause de l'acceptation tardive - la réponse n'étant entendue qu'après l'expiration du terme convenu. Dans ces deux hypothèses, les solutions proposées ne sont que l'application de la notion de l'«accord de volontés» tel que l'a conçu la jurisprudence québécoise, afin de résoudre le problème des contrats par correspondance. De plus, cette conception, loin d'être méconnue par le droit positif québécois, trouve une consécration formelle à l'art. 787 du Code civil en matière de donations. Ceci est d'autant plus remarquable si l'on considère que le Code actuel ne contient aucune disposition énonçant une règle générale quant aux contrats entre non-présents, une lacune qui a dû être suppléée par la jurisprudence en l'absence de directives législatives.

La raison principale qui milite en faveur de la reconnaissance de cette conception de l'accord de volontés comme fondement général du droit contractuel est que la notion de consensualisme est antithétique à

44. Supra, note 16, p. 288.

45. Op.cit. note 7, p. 20. 
l'idée qu'un pollicitant puisse être lié à son insu. Il faut donc consacrer, au moins en principe, la théorie de l'information.

Dans les contrats entre présents, cela ne pose guère de difficultés: il ne saurait être question d'appliquer la théorie de la réception, puisque dans cette hypothèse, réception et information coïncident. Dans les contrats entre non-présents, au contraire, certains aménagements de la théorie de l'information sont rendus nécessaires à cause de certaines considérations pratiques de preuve; d'où l'adoption de la théorie de la réception qui n'est qu'une simple présomption: l'offrant est réputé être informé de l'acceptation dès qu'il peut en prendre connaissance, i.e. dès qu'elle est en sa possession, qu'il le fasse ou non.

Appliquant ces principes au contrat conclu par téléphone, il est évident que celui-ci devrait être considéré conclu, non pas au lieu où les paroles d'acceptation sont prononcées, comme le décide la jurisprudence actuelle, mais bien au lieu où l'offrant entend ces paroles d'acceptation, car c'est seulement à ce moment qu'il y a rencontre de volontés. C'était d'ailleurs la solution retenue, à bon droit, dans l'affaire Canada Concrete Products Inc. c. Nicolet ${ }^{46}$.

\section{CONCLUSION}

La règle de base en ce qui a trait à la conclusion du contrat est donc fort simple: en toute hypothèse, nous croyons que le contrat est conclu lorsque l'offrant a une connaissance réelle ou présumée de l'acceptation du pollicité. Ce principe s'applique tant aux contrats entre présents qu'aux contrats entre non-présents. Aussi devient-il inutile de se demander si les moyens de communications sont instantanés ou non et d'appliquer aux premiers des règles différentes des seconds.

A cet égard, il est intéressant de voir si l'Avant-projet de la Loi portant réforme au Code Civil du Québec du droit des obligations aurait pour effet de modifier ce principe. L'art. 1430 se lit comme suit:

«Le contrat est conclu au lieu et au moment où l'acceptation est reçue par l'offrant, quel que soit le moyen utilisé pour la communiquer et lors même que les parties ont réservé leur accord sur certains éléments. secondaires».

46. Supra, note 35 . 
Il est évident que la première partie de cet article fut inspirée par l'art. 19 du livre V du Projet de Code Civil qui proposait que «le contrat se forme au lieu et au moment où l'offrant reçoit l'acceptation» ${ }^{47}$. Selon les commentaires de l'Office de Révision du Code Civil accompagnant le Projet de Code Civil, l'article proposé devait régir les contrats entre non-présents ainsi que les contrats par correspondance. Son but était de mettre de côté la solution retenue par la Cour suprême dans Charlebois $^{48}$.

Il y a lieu de noter d'abord que la note explicative de l'ORCC perpétue l'interprétation erronée de Charlebois voulant que cet arrêt ait édicté une solution au dilemme des contrats par correspondance basée sur les moyens de communication utilisés plutôt que sur la notion «d'agent autorisé».

En réalité, comme nous avons tenté de le démontrer, les propositions de l'ORCC et de l'Avant-projet sur les obligations ne sont que la consécration de la théorie de la réception pour les contrats entre nonprésents puisque c'est la réception par l'offrant ou par son agent expressément, implicitement ou présumément «autorisé» qui a complété l'accord de volontés. Aussi, loin d'être une solution nouvelle en droit québécois, ce que proposent le Projet de l'ORCC et l'avant-Projet ne font que confirmer ce que Charlebois avait effectivement décidé.

De plus, contrairement à ce que dit l'ORCC, cette solution ne nous semble pas devoir se restreindre aux contrats inter absentes mais devrait aussi s'appliquer au contrat entre présents.

Enfin, il faudra reconnaître à la théorie de la réception son statut véritable en droit - un pis aller pour éviter des injustices résultant du monopole que possèderait autrement le pollicitant sur la preuve de la connaissance de l'acceptation de l'offre en matière de contrats entre nonprésents. Donc, à l'exception de cet aménagement destiné à rétablir un équilibre entre les parties quant à la preuve de la conclusion d'une convention entre non-présents, la théorie applicable à la formation du contrat est celle de l'information et l'accord de volontés, dans notre droit, est plus que la simple coexistence de deux volontés qui s'ignorent.

47. L'autre partie portant sur les éléments secondaires, est tirée de l'art. 36 du livre V du Projet de Code Civil.

48. Loc.cit., note 30, p. 610. («Dans cette affaire, en effet, la Cour suprême a adopté la théorie de la réception lorsque l'offre et l'acceptation n'ont pas été communiquées par un même moyen. Lorsqu'au contraire les deux futurs contractants ont utilisé un mode de communication identique, la théorie de l'expédition subsiste. On a choisi entre ces deux théories défendables celle qui paraissait présenter le moins d'inconvénients pratiques.»). 
Comme l'a si bien dit le juge Anglin, dans l'affaire Charlebois:

«To make a contract, the law requires communication of offer and acceptance alike either to the person for whom each is respectively intended, or to his authorized agent» ${ }^{49}$.

Cette solution, avec l'élimination de la présomption voulant que l'offrant nomme comme mandataire, pour recevoir en son nom l'acceptation, la personne qu'il utilise pour faire parvenir son offre ${ }^{50}$, nous semblerait simplifier grandement le droit québécois en cette matière.

49. Supra, note 23, p. 11.

50. Voir notre note 32, supra, p. 14. 